\title{
Comparative analysis of routing protocols over WiMAX
}

\begin{abstract}
Mobile WiMAX is a fast growing broadband access technology that enables low-cost mobile Internet applications, and realizes the convergence of mobile and fixed broadband access in single air interface and network architecture. Mobile WiMAX combines Orthogonal Frequency-Division Multiple Access (OFDMA) and advanced multiple-input and multipleoutput (MIMO) schemes along with flexible bandwidth and fast link adaptation, creating a highly efficient air interface that exceeds the capacity of existing and evolving $3 \mathrm{G}$ radio access networks. WiMAX networks, built on all-IP network architecture for plug and play network deployments, can support a mix of different usage and service models. However, the End-to-End delay occurs in delivering massage for dynamic WiMAX environment. In this paper, End-To-End delay performance of the Mobile WIMAX has been studied in different situations using QulNet on two routing protocol namely Dynamic Manet on Demand (DYMO) routing Protocol and Optimized Link State (OLSR) Routing Protocol. The result shows that DYMO protocols outperform $79.5 \%$ in End to End delay better than OLSR.
\end{abstract}

Keyword: DYMO; OLSR; QualNet; WiMAX 